\title{
Introduction to Augmented Intelligence Minitrack
}

\author{
Lingyao (Ivy) Yuan \\ Iowa State University \\ lyuan@iastate.edu
}

\author{
Souren Paul \\ Northern Kentucky University \\ Souren.paul@gmail.com
}

\author{
Alan R. Dennis \\ Indiana University \\ ardennis@indiana.edu
}

\begin{abstract}
Intelligence Augmentation is defined as enhancing and elevating human's ability, intelligence, and performance through the help of information technology. Augmented intelligence is closely related to artificial intelligence. Even though the underlying technology empowering intelligence augmentation and artificial intelligence overlap, however, the goals and focuses of these two topics are fundamentally different. Intelligence
\end{abstract} augmentation put the emphasis on the human agents who use the technology and on the interactive human-machine partnership. Putting it in different words: using technology to enable humans to be better humans. Artificial intelligence, on the other hand, focuses on the development of technology (i.e. robots, avatars, chatbots, etc.) to replace human agents or perform at the level as human agents. Intelligence augmentation is at the frontier of the future for technology. Major technology companies have begun research and development of various technology to augment the work of employees to increase efficiency and improve productivity. Intelligence augmentation technologies are currently used across a number of fields, including healthcare, financial, retail, manufacturing, sales and marketing. There are a variety of important issues and topics of importance, such as the behavioral, emotional, and even physical responses of the human agents while using augmented intelligence, the underlying cognitive processes underlying the use of the technology, the impact of augmented intelligence on the firm level or industry level, and ethical issues and considerations of augmented intelligence technology. Research could be wide ranging, such as rich descriptive statistics, theories, emergent and innovative topics, models and frameworks related to technologies and their impact on marketing, case studies, methods, qualitative research, etc.

With the strong support of the HICSS Knowledge Innovation and Entrepreneurial Systems Track chairs as well as the contributing authors and quality reviewers, we have launched this minitrack for the first time this year. We positioned this minitrack as a place for researchers or practitioners from diverse background to share their research and ideas. This year, augmented intelligence minitrack has accepted two research papers.

The paper, MD-Manifold: A Medical Distance Based Manifold Learning Approach for Heart Failure Readmission Prediction, by Shaodong Wang, Qing $\mathrm{Li}$, and Wenli Zhang, proposed a new framework, Medical-Distance-Manifold (MD-Manifold) to generate low dimensional representations for patient hospital visits by combining the medical conceptstructure based distance and manifold learning. The new framework has proven to perform better and more efficient than higher-dimensional One Hot encoding and PCA. This study makes novel contribution to machine learning studies in the research area of healthcare with the potential of the new framework being applied to other areas such as mortality prediction.

The paper, Beyond deep fakes: Conceptual framework, applications, and research agenda for neural rendering of realistic digital faces, by Mike seymour, Kai Riemer, Lingyao Yuan, Alan Dennis, provides a taxonomy of the neural rendering technology and discussed the potential implications of neural rendering in different areas, in comparison with the traditional CG technology. The current neural rendering has been adopted in technologies for face swapping, deep fake, and face synthesis, in combination with or without the traditional $\mathrm{CG}$ technology. Along with the taxonomy, the authors provided four examples on the use of neural rendering in practice with ample visual support. This research has not only provided a foundation of research in the visual and graphical design area but also called for ethical and legal discussions on the develop, adoption, and promotion of such technologies. 